\title{
Parametrically Resonant Surface Waves in a Rectangular Basin
}

\section{Wu-Ting Tsai}

Associate Professor, Department of Oceanography, National Taiwan Ocean University, Keelung. Taiwan, R.O.C.

Follow this and additional works at: https://jmstt.ntou.edu.tw/journal

Part of the Oceanography and Atmospheric Sciences and Meteorology Commons

\section{Recommended Citation}

Tsai, Wu-Ting (1997) "Parametrically Resonant Surface Waves in a Rectangular Basin," Journal of Marine Science and Technology. Vol. 5: Iss. 1, Article 8.

DOI: $10.51400 / 2709-6998.2539$

Available at: https://jmstt.ntou.edu.tw/journal/vol5/iss1/8

This Research Article is brought to you for free and open access by Journal of Marine Science and Technology. It has been accepted for inclusion in Journal of Marine Science and Technology by an authorized editor of Journal of Marine Science and Technology. 


\title{
PARAMETRICALLY RESONANT SURFACE WAVES IN A RECTANGULAR BASIN
}

\author{
Wu-Ting Tsai
}

Keywords: Free-surface wave, Parametric resonance, Perturbation analysis, Nonlinear wave.

\section{ABSTRACT}

We consider the resonant motion of a layer of fluid in a twodimensional rectangular basin forced to oscillate vertically. The equation governing the slowly-varying amplitude is derived using the multiple-scales method of perturbation analysis. The solution of the evolution equation is obtained analytikally. For steady harmonic responses, the present result compares remarkably well with available experimental measurements and is an improvement over existing third-order perturbation calculations. For unsteady (periodic) motions, the periods are computed as a function of motion amplitudes. The presence of internal resonance is discussed briefly.

\section{INTRODUCTION}

The phenomenon of parametric resonance arises in a variety of water-wave problems such as cross waves in a channel, edge waves along a shoreline, and the transverse instability of progressive and standing waves. In contrast to forced oscillations, in which the forcing gives rise to an inhomogeneity in the governing equation, parametric excitations appear as coefficients of the governing differential equations.

The appearance of subharmonic standing waves in a basin forced to oscillate vertically was first observed by Faraday [1]. His experiments were repeated and the results reconfirmed by Rayleigh $[2,3]$. That parametric resonance is indeed the mechanism for the generation of such standing waves was first indicated by Benjamin and Ursell [4] who showed that the response amplitudes are governed by a Mathieu's equation. This linearized theory explains the occurrence of parametric instability at specific excitation

Paper Received March, 1997. Revised May, 1997. Accepted May, 1997. Author for Correspondence: Wu-Ting Tsai.

*Associate Professor, Department of Oceanography, National Taiwan Ocean University, Keelung, Taiwan, R.O.C. frequencies, but predicts unbounded wave amplitudesat resonance and is not able to describe the actual development of the free surface. Skalak and Yarymovych [5] and Dodge et al. [6] extended the linear analysis to higher order for the rectangular and circular basin respectively. By applying a Stokes' expansion to the velocity potentialand and the freesurface elevation, they obtained to third order finiteamplitude resonant periodic responses. Such direct perturbations in the response amplitude are however inferior in that terms of larger order of magnitude may often be omitted in the resulting equations. The analysis was improved by Ockendon and Ockendon [7] who applied multiple-scale technique to obtain an evolution equation governing the response near subharmonic resonance frequencies. Unfortunately, they did not give explicit forms of the evolution equation for specific geometries, and as a result were no comparisons to earlier perturbation analyses and experimental measurements. Recently, Miles [8] used a Lagrangian formulation and obtained, after averaging, a Hamiltonian system for the response amplitude including explicit results for the case of a circular cylinder.

In this paper, the multiple-scales expansion of Ockendon and Ockendon [7] is worked out for a twodimensional rectangular basin of arbitrary depth, and the evolution equation governing the slowlyvarying amplitude is derived explicitly. The periodic solution of the evolution equation is then obtained analytically. The results for the steady (harmonic) response amplitude compare remarkably well with the experimental data of Skalak and Yarymovych [5] and are superior to their perturbation results. Stability of the solutions around fixed points with or without the presence of a linear damping is discussed briefly with conclusions similar to that for the circular tank of Miles [8]. The possibility of internal resonance at critical depths is also indicated, but unlike the case of the circular basin, the first possible two-to-one internal resonance for this geometry is between the first symmetric and the third symmetric or ant- 
isymmetric modes which occurs as a result of cubic rather than quadratic interactions in the evolution equation.

\section{EVOLUTION EQUATION}

\section{Mathematical Formulation}

We consider the motion of an ideal, incompressible fluid under a free surface in a two-dimensional rectangular basin with a horizontal bottom subject to a vertical oscillation given by $-a_{e} \cos \left(\omega_{e} t\right)$. The resulting fluid motion is assumed to be irrotational and surface tension is ignored. A coordinate system fixed with the basin is chosen so that the origin and $x$-axis are in the undisturbed free surface and $z$ is positive upwards. The side walls of the basin are at $x= \pm L$ and the bottom at $z=-h L$. For convenience, we introduce dimensionless (primed) variables: $x^{\prime}=x / L$; $z^{\prime}=z / L ; \phi^{\prime}=\phi /\left(a_{e} \omega L\right) ; \eta^{\prime}=\eta / a_{e}$ and $t^{\prime}=\omega t$ where $\phi(x, z, t)$ is the velocity potential, $\eta(x, t)$ the free-surface elevation, and $\omega \equiv \omega_{e} / 2$ is the frequency of the resonant response. Dropping the primes hereafter, we have for the governing equation:

$$
\frac{\partial^{2} \phi}{\partial x^{2}}+\frac{\partial^{2} \phi}{\partial z^{2}}=0, \quad \text { on }-h \leq z \leq \epsilon \eta ;-1 \leq x \leq 1,(1 a)
$$

where $\epsilon \equiv a_{e} / 2$. On the side walls and bottom, the normal velocity vanishes

$$
\frac{\partial \phi}{\partial x}=0, \quad \text { on } x= \pm 1,
$$

and

$$
\frac{\partial \phi}{\partial z}=0, \quad \text { on } z=-h .
$$

On the free surface, the kinematic and dynamic boundary conditions are

$$
\frac{\partial \eta}{\partial t}+\epsilon \frac{\partial \eta}{\partial x} \frac{\partial \phi}{\partial x}-\frac{\partial \phi}{\partial z}=0
$$

on $z=\epsilon \eta(x, t),(1 d)$

and

$$
\begin{aligned}
& \frac{\partial \phi}{\partial t}+\frac{\epsilon}{2}\left[\left(\frac{\partial \phi}{\partial x}\right)^{2}+\left(\frac{\partial \phi}{\partial z}\right)^{2}\right]+v^{2}\left[N^{2} \mu+\epsilon \cos (v t)\right] \\
& \quad \text { on } z=\epsilon \eta(x, t)
\end{aligned}
$$

where $N \equiv \Omega / \omega_{e}, v \equiv \omega_{e} / \omega, \mu \equiv 1 /[(\pi \tanh (\pi h)]$, and $\Omega=$ $\left.[(\pi g / L) \tanh (\pi h)]^{1 / 2}\right\}$ is the dimensional linear natural frequency of the first (symmetric) standing wave in the basin. We consider here only the one-half subharmonic resonance of this symmetric first mode, so that $v=2$, and we write

$$
N=\frac{1}{2}+\lambda \epsilon \text {, }
$$

where $\lambda=O(1)$ is the detuning parameter.

\section{Asymptotic Analysis}

To obtain an evolution equation for the slowlyvarying amplitude of the surface, we use multiplescale analysis and introduce the slow timescale, $\tau$, which is dependent on amplitude:

$$
\tau=\epsilon t .
$$

The free-surface boundary conditions (Eqs. 1d,e) are expanded in Taylor series about $z=0$, and $\phi$ and $\eta$ are written in perturbation series:

$$
\begin{aligned}
& \phi=\epsilon^{-1 / 2} \phi_{0}+\phi_{1}+\epsilon^{1 / 2} \phi_{2}+\ldots, \\
& \eta=\epsilon^{-1 / 2} \eta_{0}+\eta_{1}+\eta^{1 / 2} \eta_{2}+\ldots
\end{aligned}
$$

We proceed, in a manner similar to Ockendon and Ockendon [7], through $O\left(\epsilon^{1 / 2}\right)$. At each order, $n$, the governing equation for $\phi_{n}$ are in the form:

$$
\begin{aligned}
& \frac{\partial^{2} \phi_{n}}{\partial x^{2}}+\frac{\partial^{2} \phi_{n}}{\partial z^{2}}=0, \quad-h \leq z \leq 0 ; 0 \leq x \leq 1, \\
& \frac{\partial \phi_{n}}{\partial x}=0, \quad x=0,1, \\
& \frac{\partial \phi_{n}}{\partial z}=0, \quad z=-h, \\
& \frac{\partial \eta_{n}}{\partial t}-\frac{\partial \phi_{n}}{\partial z}=F_{n}, \quad z=0, \\
& \frac{\partial \phi_{n}}{\partial t}+\mu \eta_{n}=G_{n}, \quad z=0,
\end{aligned}
$$

where $F_{n}$ and $G_{n}$ are given by lower-order terms.

At leading order, $F_{0}=G_{0}=0$, and the first-mode standing wave solution with slowly-varying amplitude, $A(\tau)$, is given by

$$
\begin{aligned}
& \phi_{0}=\frac{1}{2} A(\tau) e^{-i t} \cos (\pi x) \frac{\cosh \pi(z+h)}{\pi \sinh \pi h}+\underset{(6 a)}{C . C .} \\
& \eta_{0}=\frac{i}{2} A(\tau) e^{-i t} \cos (\pi x)+C . C .,
\end{aligned}
$$

where C.C. denotes the complex conjugate of the preceding term. At the next order, we obtain

$$
\begin{aligned}
& F_{1}=-\frac{\partial \eta_{0}}{\partial x} \frac{\partial \phi_{0}}{\partial x}+\eta_{0} \frac{\partial^{2} \phi_{0}}{\partial z^{2}} \\
& G_{1}=-\eta_{0} \frac{\partial^{2} \phi_{0}}{\partial t \partial z}-\frac{1}{2}\left[\left(\frac{\partial \phi_{0}}{\partial x}\right)^{2}+\left(\frac{\partial \phi_{0}}{\partial z}\right)^{2}\right]
\end{aligned}
$$


At this order, no secularity appears and we have $\phi_{1}=i A^{2} e^{-2 i t}\left[a_{0}+a_{2} \cos (2 \pi x) \frac{\cos 2 \pi(z+h)}{2 \pi \sinh 2 \pi h}\right]+C . C$.

$\eta_{1}=A^{2} e^{-2 i t} b_{2} \cos (2 \pi x)+C . C .+A A^{*}\left[c_{0}+c_{1} \cos (2 \pi x)\right],(8 b)$

where

$$
\begin{aligned}
& a_{0}=-\frac{1}{32}\left(3+\pi^{2} \mu^{2}\right), \quad a_{2}=\frac{3}{8} \pi^{2} \mu\left(\pi^{2} \mu^{2}-1\right), \\
& b_{2}=\frac{1}{16} \pi^{2} \mu\left(1-3 \pi^{2} \mu^{2}\right), \quad c_{0}=\frac{1}{8 \mu}\left(1-\pi^{2} \mu^{2}\right), \\
& c_{2}=\frac{1}{8 \mu}\left(1+\pi^{2} \mu^{2}\right) .
\end{aligned}
$$

At order $\epsilon^{1 / 2}, F_{2}$ and, $G_{2}$ are given by

$$
\begin{gathered}
F_{2}=-\frac{\partial \eta_{0}}{\partial \tau}-\frac{\partial \eta_{0}}{\partial x} \frac{\partial \phi_{1}}{\partial x}-\frac{\partial \eta_{1}}{\partial x} \frac{\partial \phi_{0}}{\partial x}+\eta_{0} \frac{\partial^{2} \phi_{1}}{\partial z^{2}} \\
+\eta_{1} \frac{\partial^{2} \phi_{0}}{\partial z^{2}}-\eta_{0} \frac{\partial \eta_{0}}{\partial x} \frac{\partial^{2} \phi_{0}}{\partial x \partial z}+\frac{1}{2} \eta_{0}^{2} \frac{\partial^{3} \phi_{0}}{\partial z^{3}} \\
G_{2}=-\frac{\partial \phi}{\partial \tau}-\eta_{0} \frac{\partial^{2} \phi_{1}}{\partial t \partial z}-\eta_{1} \frac{\partial^{2} \phi_{0}}{\partial t \partial z}-\frac{1}{2} \eta_{0}^{2} \frac{\partial^{3} \phi_{0}}{\partial t \partial z^{2}} \\
-\frac{\partial \phi_{0}}{\partial x} \frac{\partial \phi_{1}}{\partial x}-\frac{\partial \phi_{0}}{\partial z} \frac{\partial \phi_{1}}{\partial z}-\eta_{0} \frac{\partial \phi_{0}}{\partial x} \frac{\partial^{2} \phi_{0}}{\partial x \partial z} \\
-\eta_{0} \frac{\partial \phi_{0}}{\partial z} \frac{\partial^{2} \phi_{0}}{\partial z^{2}}-4(\mu \lambda+\cos 2 t) \eta_{0}
\end{gathered}
$$

Substituting $\phi_{0}, \phi_{1}, \eta_{0}$ and $\eta_{1}$ into Eqs. $(9 a)$ and $(9 b)$, and eliminating $\eta_{2}$, we obtain the free-surface boundary condition for $\phi_{2}$ :

$$
\begin{aligned}
\frac{\partial^{2} \phi_{2}}{\partial t^{2}}+\mu \frac{\partial \phi_{2}}{\partial z} & =\left(i \mu \frac{d A}{d \tau}+A^{*}-2 \mu \lambda A\right. \\
& \left.+\Gamma A^{2} A^{*}\right) e^{-i t} \cos (\pi x)+C . C . \\
& + \text { nonsecular terms +higher modes in } \\
& x, \quad(10 a)
\end{aligned}
$$

where

$$
\Gamma \equiv \frac{1}{64 \mu}\left(6-5 \mu^{2} \pi^{2}+16 \mu^{4} \pi^{4}-9 \mu^{6} \pi^{6}\right) .
$$

To avoid secularity in Eq. (10a), we require

$$
\mu \frac{d A}{d \tau}+2 i \mu \lambda A-i A^{*}-i \Gamma A^{2} A^{*}=0,
$$

which is the governing equation for the slowly-varying complex amplitude $A(\tau)$. If we let $A(\tau) \equiv C(\tau)$ $+i D(\tau)$, where $C$ and $D$ are real amplitudes, the evolution equation Eq. (11) can be rewritten as

$$
\mu \frac{d C}{d \tau}-D\left[(1+2 \mu \lambda)-\Gamma\left(C^{2}+D^{2}\right)\right]=0,
$$

and

$$
\mu \frac{d D}{d \tau}-C\left[(1-2 \mu \lambda)+\Gamma\left(C^{2}+D^{2}\right)\right]=0 .
$$

\section{Phase-Plane Analysis}

The phase-plane analysis of Eq. (12) has been given in Ockendon and Ockendon [7] and Miles [8]. For the sake of later discussions, the solution for $h=\infty$ and $\mu \lambda=-1,0,1$ respectively are shown in Fig. 1 . Note that for $\mu \lambda<-1 / 2$, the only critical point is a stable center at $C_{0}=0, D_{0}=0$. For $|\mu \lambda|<-1 / 2, C=0, D=0$ becomes an unstable saddle point and the stable points are at $C_{0}=0, D_{0}= \pm[(2 \mu \lambda+1) / \Gamma]^{1 / 2}$. The equations of the two separatrices are

$$
C^{2}+D^{2} \pm 2\left[\frac{C D-\mu \lambda\left(C^{2}+D^{2}\right)}{\Gamma}\right]^{1 / 2}=0 .
$$

For $\mu \lambda>1 / 2$, there are thre estable centers: $C_{0}=0, D_{0}=0$ and $C_{0}=0, D_{0}= \pm[(2 \mu \lambda+1) / \Gamma]^{1 / 2}$; and two unstable saddle points: $C_{0}= \pm[(2 \mu \lambda-1) / \Gamma]^{1 / 2}, D_{0}=0$. For this case, the two separatrices are the circles given by

$$
C^{2}+\left(D^{2} \pm \frac{1}{\sqrt{\Gamma}}\right)^{2}=\frac{2 \mu \lambda}{\Gamma} .
$$

\section{Weakly Damped System}

If a weak, linear damping is present in the dy-
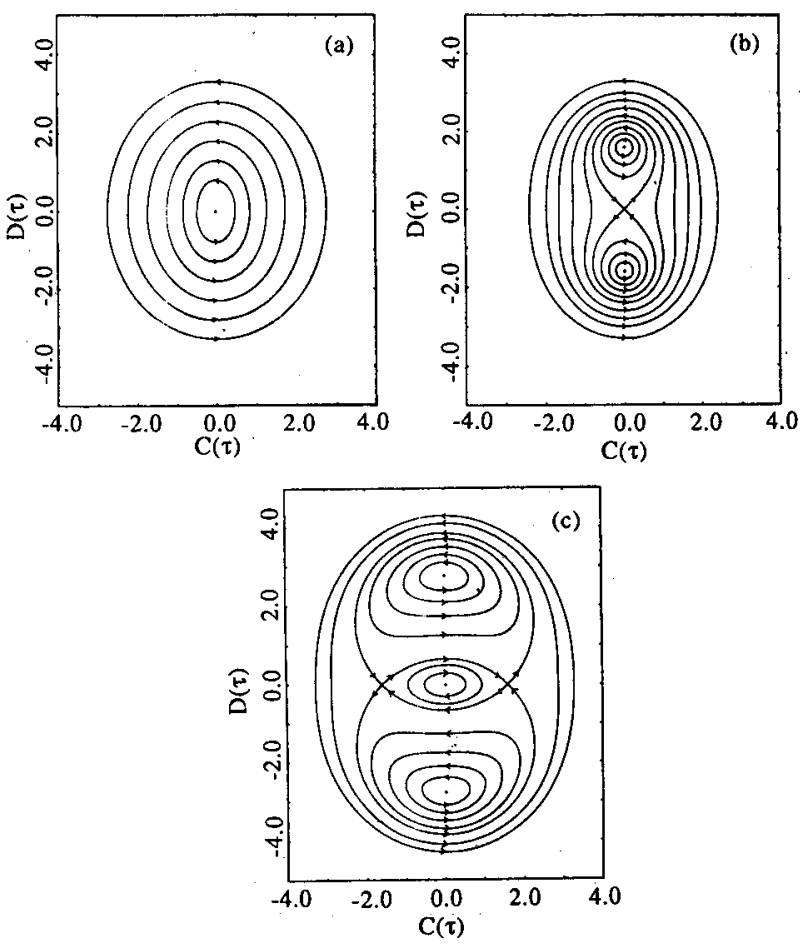

Fig. 1. Phase plane solution of the undamped evolution equation (11) for $h=\infty$, and (a) $\mu \lambda=-1$, (b) $\mu \lambda=0$, and (c) $\mu \lambda=1$. 
namic system (as in Miles [8]), the evolution equation Eq. (11) is simply

$$
\mu \frac{d A}{d \tau}+\alpha A+2 i \mu \lambda A-i A^{*}-i \Gamma A^{2} A^{*}=0,
$$

where $\alpha=\delta / \epsilon$, and $\delta$ is the ratio of the actual to the critical damping of the free oscillation of the resonant mode. In the damped system Eq. (13), the stable points in the phase-plane become asymptotically stable spirals, while the unstable saddle points remain unchanged. For $\mu \lambda<-\delta / 2, C_{0}=0, D_{0}=0$ is a stable spiral point. For $|\mu \lambda|<\delta / 2, C_{0}=0, D_{0}=0$ becomes an unstable saddle point, and $C_{0}= \pm[(2 \mu \lambda+\delta)$ $(1-\delta) /(2 \Gamma)]^{1 / 2}, D_{0}= \pm[(2 \mu \lambda+\delta)(1+\delta) /(2 \Gamma)]^{1 / 2}$, are the stable spiral points. For $\mu \lambda>\delta / 2, C_{0}=0, D_{0}=0$ and $C_{0}= \pm[(2 \mu \lambda+\delta)(1-\delta) /(2 \Gamma)]^{1 / 2}, D_{0}= \pm[(2 \mu \lambda+\delta)(1+\delta) /$ $(2 \Gamma)]^{1 / 2}$, are the three stable spiral points, while $C_{0}= \pm[(2 \mu \lambda-\delta)(1+\delta) /(2 \Gamma)]^{1 / 2}, D_{0}= \pm[(2 \mu \lambda-\delta)(1-\delta) /$ $(2 \Gamma)]^{1 / 2}$, are the two unstable saddle points. The phase-plane diagram for the case $\alpha=0.5, h=\infty$ and $\mu \lambda=-1,0,1$ respectively arepresented in Fig. 2 for comparison.

\section{SOLUTION OF THE EVOLUTION EQUATION}

Periodic solutions of the undamped evolution equation Eq. (11) can be obtained analytically (see e.g. Struble [9]). Representing the complex amplitude $A(\tau)$ as

$$
A(\tau)=C(\tau)+i D(\tau) \equiv a(\cos \gamma+i \sin \gamma),
$$

where $a$ and $\gamma$ are functions of $\tau$. Equation (11) can now be written as

$$
\begin{aligned}
& \mu \frac{d \gamma}{d \tau}=-2 \mu \lambda+\Gamma a^{2}+\cos 2 \gamma, \\
& \mu \frac{d a}{d \tau}=a \sin 2 \gamma .
\end{aligned}
$$

Combining Eqs. (15a) and (15b) and eliminating $\tau$, we obtain

$$
\frac{d \gamma}{d a}=\frac{\cos 2 \gamma-2 \mu \lambda+\Gamma a^{2}}{a \sin 2 \gamma} .
$$

Equation (16) is an exact integral and upon integration we have

$$
2 a^{2}(\cos 2 \gamma-2 \mu \lambda)+\Gamma a^{4}=E
$$

where $E$ is an integration constant. Equations (15b) and (17) can be further combined as

$$
\frac{\mu}{a} \frac{d a}{d \tau}= \pm\left[1-\left[2 \mu \lambda+\left(E-\Gamma a^{4}\right) /\left(2 a^{2}\right)\right]^{2}\right]^{1 / 2},
$$

which gives

$$
\tau= \pm \frac{\mu}{2} \int \frac{d a^{2}}{\left[a^{4}-\left(E / 2+2 \mu \lambda a^{2}-\Gamma a^{4} / 2\right)^{2}\right]^{1 / 2}}
$$

Thus the slow time $\tau$ is expressed as an elliptic integral of the square of the amplitude $a$. At any specified $\tau, a^{2}$ is given in terms of an elliptic function of $\tau$, and the phase angle $\gamma$ can be obtained from Eq. (17).

The period of the modulation, $T$, may be expressed in a more explicit form. For simplicity, we classify the phase-plane trajectories into three different types:

(i) All the trajectories in Figs. (1a) and (1b) which are outside the separatrix, and the trajectories in Fig. (1c) which are outside the trajectory with $E=0$ (this trajectories is outside the separatrix). Each of these trajectories has a different positive integration constant $E$.

(ii) Trajectories in Figs. (1b) and (1c) which are inside the upper and lower separatrices forming two nested sets about the centers $C_{0}=0, D_{0}= \pm$ $[(2 \mu \lambda+1) / \Gamma]^{1 / 2}$. Each pair of trajectories which are symmetric about the $C$ axis share the same negative $E$ value.
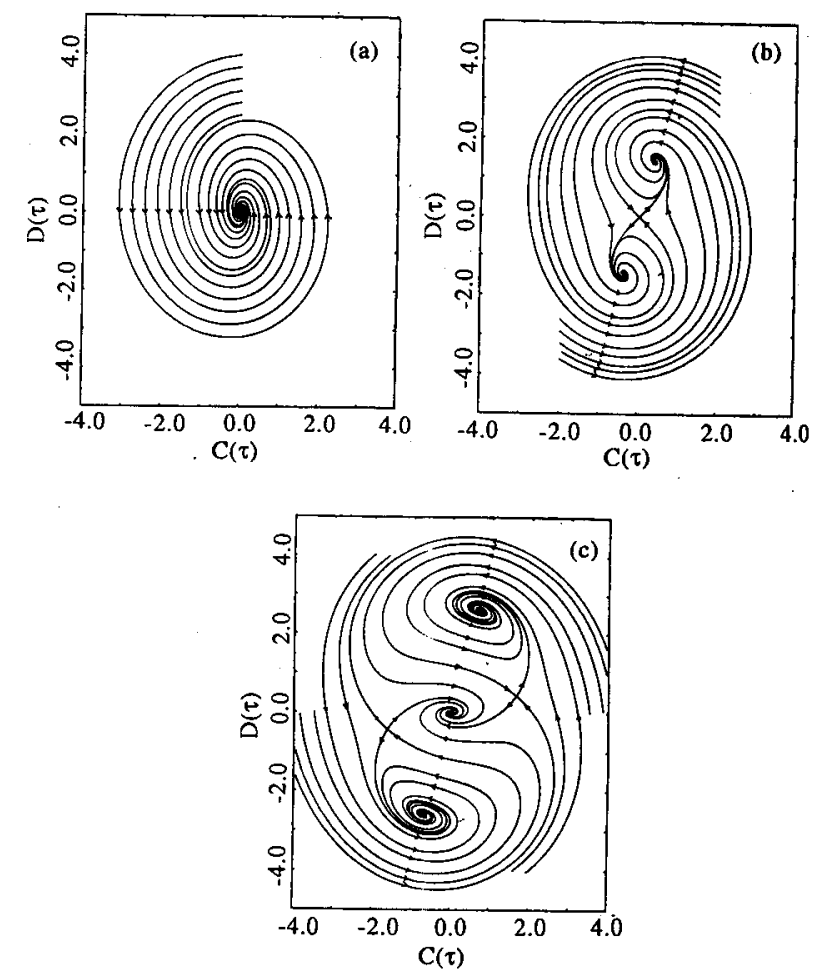

Fig. 2. Phase plane solution of the linearly-damped evolution equation (13) for $\delta=0.5, h=\infty$, and (a) $\mu \lambda=-1$, (b) $\mu \lambda=-1$, and (c) $\mu \lambda=1$. 
(iii) Trajectories in Fig. (1c) which are inside the inner separatrix and those which are outside the upper and lower separatrices but inside the $E=0$ trajectory, forming two nested sets about the stable center $C_{0}=0, D_{0}=0$. For each trajectory in the first set with a given negative integration constant $E$, there is a trajectory of the other set sharing the same constant. We rewrite Eq. (18) in the form:

$$
\tau= \pm \frac{\mu}{T} \int \frac{d s}{\left[\left(s_{1}-s\right)\left(s-s_{2}\right)\left(s-s_{3}\right)\left(s-s_{4}\right)\right]^{1 / 2}},
$$

where $s \equiv a^{2}$, and $s_{1}, s_{2}, s_{3}, s_{4}$ are the zeros of the denominator in Eq. (18).

For type (i) trajectories, only two of the zeros are real, which correspond to the intercepts of the trajectories with the $C$ and $D$ axes according to Eq. (17). Form Eq. (19), the period for type (i) trajectories is given by

$$
T=\frac{4 \mu}{\Gamma} \int_{a_{m}^{2}}^{a_{M}^{2}} \frac{d s}{\left\{\left(a_{M}^{2}-s\right)\left(s-a_{m}^{2}\right)\left[\left(s-b_{1}\right)+a_{1}^{2}\right]\right\}^{1 / 2}},
$$

where $a_{M}$ and $a_{m}$ are respectively the maximum and minimum of the amplitude, and

$$
\begin{aligned}
& a_{1}^{2}=\frac{E^{2}}{\Gamma^{2} a_{M}^{2} a_{m}^{2}}-\left[\frac{4 \mu \lambda}{\Gamma}-\frac{1}{2}\left(a_{M}^{2}+a_{m}^{2}\right)\right]^{2}, \\
& b_{1}=\frac{4 \mu \lambda}{\Gamma}-\frac{1}{2}\left(a_{M}^{2}+a_{m}^{2}\right) .
\end{aligned}
$$

Equations (20) can be further reduced to the standard form of a complete elliptic integral (see e.g. Byrd and Friedman [10]):

$$
T=\frac{4 \mu}{\Gamma} g \int_{0}^{\pi / 2} \frac{d \theta}{\left(1-k^{2} \sin ^{2} \theta\right)^{1 / 2}}=\frac{2 \mu_{g} K\left(k^{2}\right)}{\Gamma},
$$

where

$$
\begin{aligned}
& g=\frac{2}{\sqrt{A B}}, \\
& k=\frac{1}{2} \sqrt{\frac{a_{M}^{2}-a_{m}^{2}-(A-B)^{2}}{A B},} \\
& A=\sqrt{\left(a_{M}^{2}-b_{1}\right)^{2}+a_{1}^{2}}, \\
& B=\sqrt{\left(a^{2}-b_{1}\right)^{2}+a_{1}^{2}},
\end{aligned}
$$

and $K$ is the complete elliptic integral of the first kind (see Abramowitz and Stegun [11]). For type (ii) modulation, the periods of the upper and lower loops are equal and given by half times the expression Eq. $(21 a)$.

For type (iii) trajectories, the inner and outer loops associated with the same constant $E$ have equal period even though the motions are quite different. From Eq. (19), the period $T$ can be represented as

$$
T=\frac{4 \mu}{\Gamma} \int_{a_{m}^{2}}^{a_{M}^{2}} \frac{d s}{\left[\left(\bar{a}_{M}^{2}-s\right)\left(\bar{a}_{m}^{2}-s\right)\left(s-a_{m}^{2}\right)\right]^{1 / 2}},
$$

where $\bar{a}_{M}$ and $\bar{a}_{m}$ are respectively the maximum and minimum of the outer trajectory, and $a_{M}$ and $a_{m}$ the corresponding values for the inner trajectories. $\mathrm{Re}$ ducing to standard form, Eq. (22) becomes

$$
T=\frac{4 \mu g K\left(k^{2}\right)}{\Gamma},
$$

where

$$
\begin{aligned}
& g=\frac{2}{\left[\left(\bar{a}_{M}^{2}-a_{M}^{2}\right)\left(\bar{a}_{m}^{2}-a_{m}^{2}\right)\right]^{1 / 2}}, \\
& k=\left[\frac{\left(\bar{a}_{M}^{2}-\bar{a}_{m}^{2}\right)\left(a_{M}^{2}-a_{m}^{2}\right)}{\left(\bar{a}_{M}^{2}-a_{M}^{2}\right)\left(\bar{a}_{m}^{2}-a_{m}^{2}\right)}\right]^{1 / 2} .
\end{aligned}
$$

The preceding results for the period of the slowly-varying modulations are plotted in Fig. 3 as a function of the amplitude on the positive $D$ axis for $h=\infty$ and $\mu \lambda=-1,0,1$ respectively. Note that the periods are discontinuous across the separatrices and saddle points.

\section{DISCUSSION}

\section{Steady Response}

For the steady (harmonic) response, the response amplitude $z_{0}$ is defined as the maximum vertical distance between the trough and the crest of the free surface. Thus $z_{0}$ is given by twice the amplitude of $A$ at the critical points

$$
z_{0}=2\left\{\begin{array}{l}
D_{0} \\
C_{0}
\end{array}\right\}=2\left[\frac{(2 \mu \lambda \pm 1)}{\Gamma}\right],
$$

for $\mu \lambda> \begin{cases}-1 / 2, & \text { stable response, } \\ 1 / 2, & \text { unstable response. }\end{cases}$

For deep water, $\Gamma=\pi / 8$, and the values for $z_{0}$ are 
compared to the third-order perturbation results and experimental data of Skalak and Yarymovych [5] in Fig. 4, where $\sigma \equiv \omega / \Omega$. The experiments of Skalak and Yarymovych were conducted in a vertically vibrating tank with a 2 inches $\times 6$ inches rectangular cross section and 18 inches depth. The tank was filled with tap water to a depth of 12 inches. Measurements were taken for the second harmonic mode with a wavelength of 6 inches. The amplitude of the vertical excitation of the tank was fixed at $0.3 /(2 \pi)$ inches. The comparison between the present result and the measurements is remarkable close and is better than that of the analytical results of Skalak and Yarymovych. The perturbation analysis of Skalak and Yarymovych was based on the method of Penney and Price [12] for free standing waves. The major difference between the present asymptotic analysis and that of Skalak and Yarymovych is in the assumption of the order of magnitude in performing the perturbation expansion. For the present analysis, given a vertical excitation of $O(\epsilon)$, the leading order of magnitude of the resonantly excited wave motion is $O(\epsilon \phi)=\epsilon^{1 / 2}$. The analysis of Skalak and Yarymovych is equivalent to assuming an $O(\epsilon)$ of excited wave motion in our perturbation analysis. The measured amplitudes of excited surface motions appeared to be with order of magnitude lower than that of the forced excitation. This explains why the present theoretical results are superior to that of Skalak and

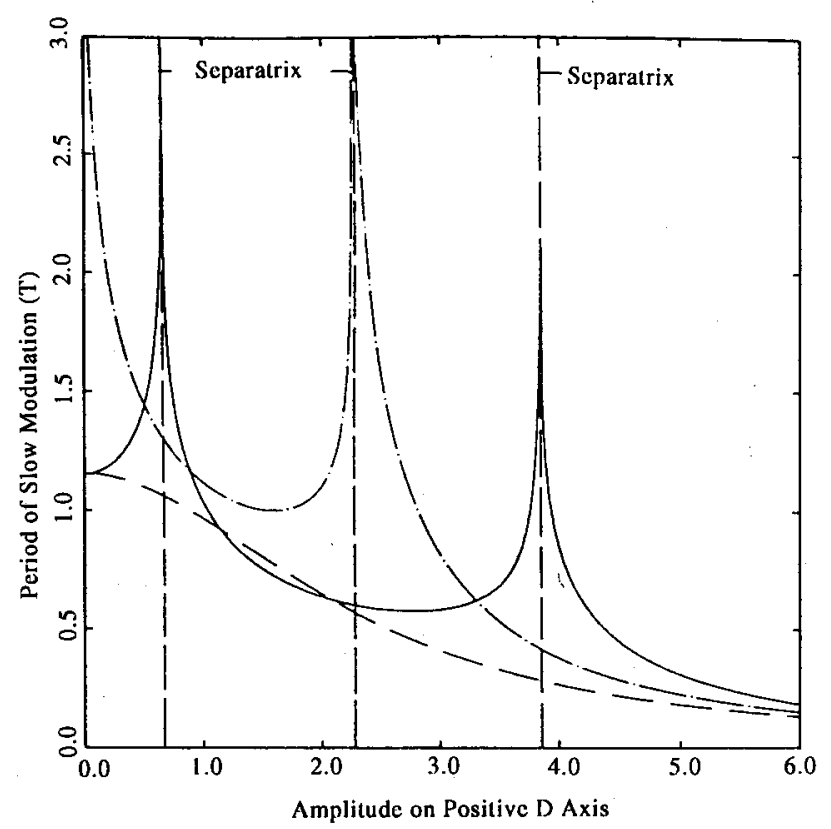

Fig. 3. Period of the slowly-varying modulation of the undamped evolution equation (11) for $h=\infty$ and $\mu \lambda=-1(-), \mu \lambda=0(-.-)$, and $\mu \lambda=1(-)$.
Yarymovych in comparing with the experimental measurements.

\section{Critical Depth $h^{*}$}

From Eq. $(10 b)$ we see that $\Gamma=0$ when the depth to length ratio $h$ satisfies

$$
6 \tanh ^{6} \pi h-5 \tanh ^{4} \pi h+16 \tanh ^{2} \pi h-9=0,
$$

which has a root at $\pi h \equiv \pi h^{*} \cong 1.022$, and $\Gamma>(<) 0$ for $h>(<) h^{*}$. Thus the harmonic response will increase/ decrease with increasing detuning $\mu \lambda$ for depth $h$ greater/less than $h^{*}$. In the neighborhood of $h^{*}$, the preceding analysis is inadequate, and higher-order terms in $\epsilon$ must be retained. At the critical depth $h=h^{*}$, the perturbation analysis above breaks down. To obtain a uniformly valid description for $h$ near $h^{*}, \phi$ and $\eta$ need to be expanded in powers of $\epsilon^{1 / 4}$, and the perturbation analysis is carried out to the fifth order.

The presence of a similar depth has also been observed for two-dimensional free standing waves by Tadjbakhsh and Keller [13] in their third-order perturbation result. From their equation (35), we find that the free standing wave frequency coincides with the linear frequency at a depth given by

$$
2 \tanh ^{6} \pi h+3 \tanh ^{4} \pi h+12 \tanh ^{2} \pi h-9=0
$$

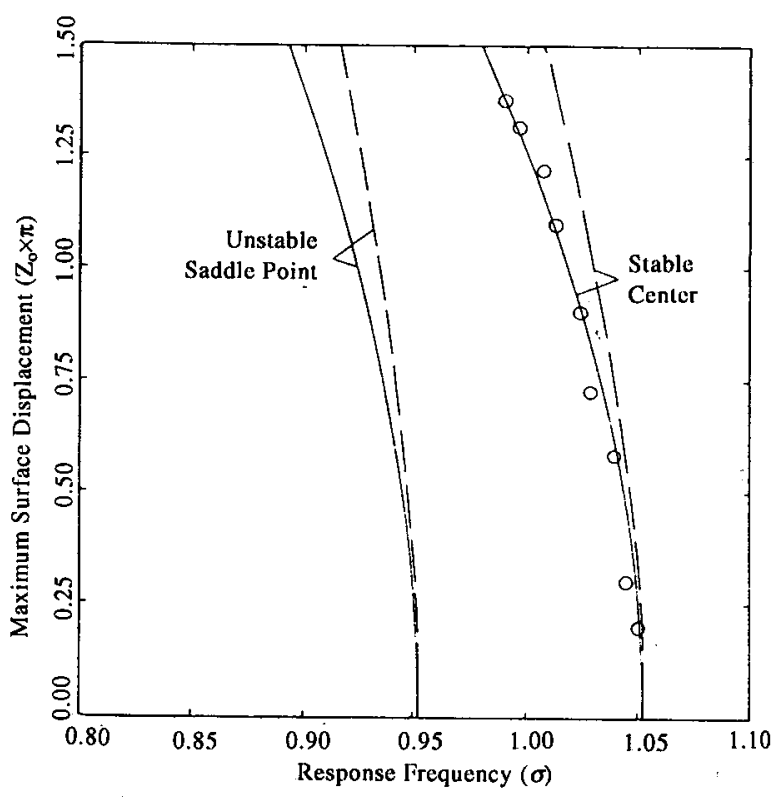

Fig. 4. Comparisons between the present theory (-) and third-order perturbation results $\left(-\right.$ ) and experimental measurements $\left({ }^{\circ}\right.$ ) of Skalak and Yarymovych [5] for the harmonic response as a function of the response frequency $\sigma \equiv \omega / \Omega$. 
which has a solution $\cong 1.058$ (this is more accurate than their $\pi h^{* *} \cong 1.07$ ). For depths greater/less than $h^{* *}$, the standing-wave frequency decreases/increases with increasing amplitude corresponding to a softening/hardening spring. (There are no physical relation between $h^{*}$ and $h^{* *}$, which are critical depths arise in the perturbation analysis of a two-dimensional "parametrically-excited" standing wave and a "freeoscillatory" standing wave respectively. Similar critical depth would also appear in the asymptotic analysis of the two-dimensional standing waves generated by other excitation mechanisms.)

\section{Internal Resonance}

As noted by Tadjbakhsh and Keller [13] for two-dimensional standing waves, and by Mack [14] for axisymmetric standing waves, an unique solution does not exist at critical values of the fluid depth for which the standing wave frequency from linearized theory is an integral multiple of the fundamental frequency, and the amplitude of a higher mode becomes comparable to that of the fundamental mode. This is also true for waves which are parametrically excited. Near these particular depths, internal resonance occurs between the parametrically-excited dominant frequency. For a circular basin, Miles [8] analyzed the two-to-one (i.e., $\omega_{2}=2 \omega_{1}$ ) internal resonance between the parametrically resonant first antisymmetric mode and the first axisymmetric mode. By assuming that the internal resonance is perfectly tuned, Miles found that every steady-state response is either trivial or harmonic. Nayfeh [15] reexamined this problem but relaxed the perfectlytuned assumption for internal resonance. For sufficiently large values of detuning, he found Hopf bifurcations, and hence aperiodic steady-state responses.

Unlike the resonant waves in a circular tank, the first possible two-to-one internal resonance in a two-dimensional rectangular basin is between the first symmetric and the third symmetric or antisymmetric mode. Assuming the detuning parameter for the $q$ th internally resonant mode be

$$
\frac{\Omega_{q}}{\omega_{e}}=1+\lambda_{q} \epsilon,
$$

where $\Omega_{q}$ is the dimensional linear natural frequency of the $q$ th mode, and following a similar procedure as before, we obtain the equations governing the evolution of the complex amplitudes of the dominant (first) mode $A(\tau)$ and that of the $q$ th mode $A_{q}(\tau)$ :

$$
\mu \frac{d A}{d \tau}+2 i \mu \lambda A-i A^{*}-i \Gamma A^{2} A^{*}-i A A_{q}^{2} \Sigma=0,
$$

$$
2 \mu_{q} \frac{d A}{d \tau}+8 i \mu_{q} \lambda_{q} A_{q}-i A^{*}-i \Gamma_{q} A_{q}^{2} A_{q}^{*}-i A_{q} A_{q}^{2} \Sigma_{q}=0
$$

where $\mu_{q}=1 /[q \pi \tanh (q \pi h)]$ and $\Gamma, \Sigma, \Gamma_{q}, \Sigma_{q}$ are functions of $q$ and $h$. Thus, for the two-dimensional rectangular tank, the nonlinear coupling interaction between two internal resonant modes are cubic, rather than quadratic as in the case of the circular basin analyzed by Miles [8] and Nayfeh [15].

\section{CONCLUSION}

Parametrically excited surface wave in a twodimensional rectangular tank under vertical oscillation was studied. The evolution equation, governing the slowly-varying amplitude, was first derived using the method of multiple scales. Linear stability analysis around the stationary solutions of the evolution equation was conducted. The complete solutions (on the whole phase plane) of the evolution equation were obtained analytically. For steady harmonic responses, the present result compares remarkably well with available experimental measurements (by Skalak and Yarymovych [5]) and is an improvement over the third-order perturbation calculations of Skalak and Yarymovych. This is attributed to better scaling (ordering) of the present perturbation analysis than that of Skalak and Yarymovych.

\section{REFERENCES}

1. Faraday, M., "On the Forms and States Assumed by Fluids in Contact with Vibrating Elastic Surfaces," Phil. Trans. Roy. Soc. Lond. A, Vol. 121, pp. 319-340 (1831).

2. Rayleigh, Lord, "On Maintained Vibrations," Phil. Mag., Vol. 15, pp. 229-235 (1883).

3. Rayleigh, Lord,"On the Crispations of Fluid Resting upon a Vibrating Support." Phil. Mag., Vol. 16, pp. 50-58 (1883).

4. Benjamin, T.B. and Ursell, F., "The Stability of The Plane Free Surface of a Liquid in Vertical Periodic Motion," Proc. Roy. Soc. Lond. A, Vol. 225, pp. 505515 (1954).

5. Skalak, R. and Yarymovych, M.I., "Forced Large Amplitude Surface Waves," Proc. 4th U.S. Nat. Cong. Appl. Mech., pp. 1411-1418 (1962).

6. Dodge, F.T., Kana, D.D. and Abramson, N., "Liquid Surface Oscillations in Longitudinally Excited rigid Cylindrical Containers," AIAA J., Vol. 3, pp. 685-695 (1965).

7. Ockendon, J.B. and Ockendon, H., "Resonant Surface Waves," J. Fluid Mech., Vol. 59, pp. 397-413 
(1973).

8. Miles, J.N., "Nonlinear Faraday Resonance," J. Fluid Mech., Vol. 146, pp. 285-302 (1984).

9. Struble, R.A., "Oscillations of a Pendulum Under Parametric Excitation,"Quart. Appl. Math., Vol. 21, pp. 121-131 (1963).

10. Byrd, P.F. and Friedman, M.D., Handbook of Elliptic Integrals for Physicists and Engineers, SpringerVerlag, Berlin (1954).

11. Abramowitz, M. and Stegun, I.A., Handbook of Mathematical Functions, Dover, New York (1965).

12. Penney, W.G. and Price, A.T., "Some Gravity Wave Problems in the Motion of Perfect Liquids, Part II, Finite Periodic Stationary Gravity Waves in a Perfect Liquid," Phil. Trans. R. Soc. Lond., Vol. A244, pp. 254-268 (1952).

13. Tadjbakhsh, I. and Keller, J.B., "Standing Surface Waves of Finite Amplitude," J. Fluid Mech., Vol. 8, pp. 442-451 (1960).

14. Mack, L.R., "Periodic, Finite-Amplitude, Axisymmetric Gravity Waves," J. Geophys. Res., Vol. 67, pp. 829-843 (1962).

15. Nayfeh, A.H., "Surface Waves in Closed Basins Un- der Parametric and Internal resonances," Phys. Fluids, Vol. 30, pp. 2976-2983 (1987).

\section{垂直振蕰水槽中之共振波動}

蔡武廷

國立台海海洋大掌海洋科量系

摘 要

本文探討強制垂直振動之二維矩形水槽中之 自由液面因共振而産生的波動。利用多尺度微摄分 析我們推琶了波動振幅之演化引導方程式。同時我 們亦求得振幅運動演化方程式之解析解, 將振幅演 化之用期表示成振幅之函数。由振幅演化方程式之 定常解, 我們可得到波動之简諧運動振幅, 其理論 結果與㝜验值非常之吻合。我們亦討論可能發生之 內共振, 以及在特殊水深微摄分析座生奇異之情 㫛。 